\title{
A Framework for Low-Power IPv6 Routing Simulation, Experimentation, and Evaluation
}

\author{
Nicolas Tsiftes, Joakim Eriksson, Niclas Finne, Fredrik Österlind, Joel Höglund, Adam Dunkels \\ Swedish Institute of Computer Science \\ \{nvt,joakime,nfi,fros,joel,adam\}@sics.se
}

\begin{abstract}
Low-power networked devices, such as sensors and actuators, are becoming a vital part of our everyday infrastructure. Being networked, the continued development of these systems needs involvement from the networking community. We present a framework for simulation, experimentation, and evaluation of routing mechanisms for low-power IPv6 networking. The framework provides a detailed simulation environment for low-power routing mechanisms, and allows the system to be directly uploaded to a physical testbed for experimental measurements.
\end{abstract}

\section{Categories and Subject Descriptors}

C.2.2 [Network Protocols]: Routing protocols

\section{General Terms}

Experimentation, Measurement, Performance

\section{INTRODUCTION}

The use of low-power networked devices, such as batterypowered sensors and actuators, are emerging in many areas from industrial automation and home automation to automated electric meter reading and the smart grid. Companies in the network industry such as Cisco and Ericsson predict that these networks will grow to billions of devices over the next ten years. With low-power networked devices becoming a vital part of our everyday infrastructure, it is important that the networking community gets involved in developing and evaluating network mechanisms for these devices.

To leverage existing systems and protocols for the emerging low-power networked devices, the community has adopted IP as the network platform. To allow for a large number of devices, IPv6 has been selected over IPv4. Recent work in the wireless sensor network community has shown that IPv6 can be used even for challenging and severely power and memory constrained wireless networks $[1,2]$. To achieve lowpower operation, nodes must keep their radio transceivers off as much as possible, while turning them on often enough for the nodes to be able to forward traffic. Low-power operation is achieved by a duty-cycling mechanism running below the IP layer.

Industrial adoption requires standardization, and to standardize routing for low-power and lossy IPv6 networks, the

Copyright is held by the author/owner(s).

SIGCOMM'10, August 30-September 3, 2010, New Delhi, India.

ACM 978-1-4503-0201-2/10/08.

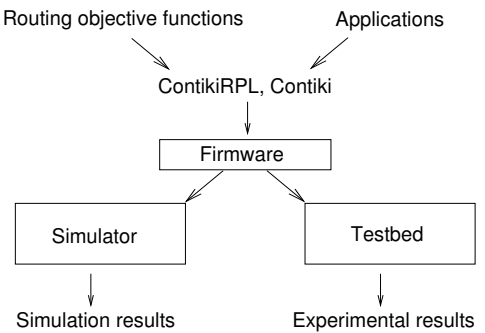

Figure 1: Workflow: a user-supplied routing objective function is compiled with the Contiki operating system, ContikiRPL, and various applications. The resulting system firmware can be simulated as well as run on real hardware.

IETF ROLL working group has developed a routing protocol called RPL $[3,4]$. Since the emerging low-power networks have vastly varying requirements and characteristics, RPL does not define any specific routing metrics, path costs, or forwarding policies. Instead, RPL leaves this open so that different networks can apply different mechanisms to meet different objectives such as minimizing latency or minimizing energy consumption. How to select forwarding policies, path costs, and routing metrics to meet specific requirements is still an open problem, however.

\section{FRAMEWORK}

We present a framework for simulation, experimentation, and evaluation of routing mechanisms in networks of lowpower devices. Our framework consist of three parts: our Contiki operating system, running on the individual sensor nodes; our Cooja/MSPsim Contiki network simulation, which allows cycle-accurate emulation of node software and bit-level accurate simulation of low-power radio networking; and our ContikiRPL implementation of the RPL protocol, which allows replaceable routing objective functions. Parts of our framework are already widely used in the industry, such as the Contiki operating system and its $\mu$ IPv6 IP stack.

The workflow of the system is that routing objective functions can first be implemented and studied in the simulator, then be transferred to a testbed with actual sensor network motes. This procedure is illustrated in Figure 1.

Our framework allows routing mechanisms both to be studied in simulation and to be immediately transferred to a physical testbed for experimental measurement and analysis. The simulation includes node-level details such as the 
effects of transceiver duty cycling, thus allowing the user to gain insight into low-level phenomena that affect the realworld performance. The experimental system provides a software-based power profiling mechanism for power measurements. Such a workflow is not possible with traditional network simulators such as ns- 2 or Omnet++, which require routing mechanisms to be developed explicitly for the simulation environment. Similarly, traditional wireless sensor network simulators such as the TinyOS TOSSIM do not provide the level of simulation detail to capture the effects of radio duty cycling.

\subsection{Node Operating System}

Our framework uses the Contiki operating system, a widely used operating system for low-power wireless devices. Contiki contains the $\mu \mathrm{IPv} 6$, a full IPv6 stack that has been certified under the IPv6 Ready program [1]. $\mu \mathrm{IPv} 6$ includes a comprehensive API for programming protocols using UDP, TCP, or ICMPv6. To achieve low-power operation, Contiki provides a set of radio duty cycling mechanisms including variants of Low-Power Listening (LPL) and Low-Power Probing (LPP).

\subsection{Routing with ContikiRPL}

ContikiRPL is the main IPv6 routing protocol in Contiki. RPL is a distance-vector protocol for IPv6 networks comprising low-power devices connected by lossy links. The protocol maintains Directed Acyclic Graph (DAG) topologies toward root nodes. The topologies are built proactively according to an objective function. Since RPL must be able to operate in multiple types of deployments and link-layers, it is flexible regarding the rules to form topologies and to select next-hops for individual packets. Routing decisions are taken by the objective function, which essentially specifies the constraints and metrics used in a network.

The chief goal of the ContikiRPL design is to provide a versatile yet simple programming interface that can be used by researchers to study objective functions. While the logic of how to exchange routing control messages has largely been specified in Internet drafts published by the IETF, objective functions are a relatively unexplored area that requires input from the research community.

\subsection{Network Simulator}

The Cooja/MSPsim network simulation provides cycleaccurate simulation of the individual devices, as well as bitlevel accurate simulation of the devices' radio transceivers. This is important since it makes it possible to run the exact same binaries in the simulator as on actual hardware. The simulator provides a set of radio models for simulating at different levels of detail.

The simulator provides a set of visualizer modules. Figure 2 shows a network visualizer and a timeline visualizer. The network visualizer shows the paths selected by the routing protocol, as well as routing statistics for each node. The timeline shows when the devices' radio transceivers are turned on or off, allowing the user to visually inspect the lowpower operation of the system, and the implications thereof such as collisions.

\section{DEMONSTRATION}

We demonstrate four parts of our system: the simulation environment and its visualizers, the low-power IPv6 oper-

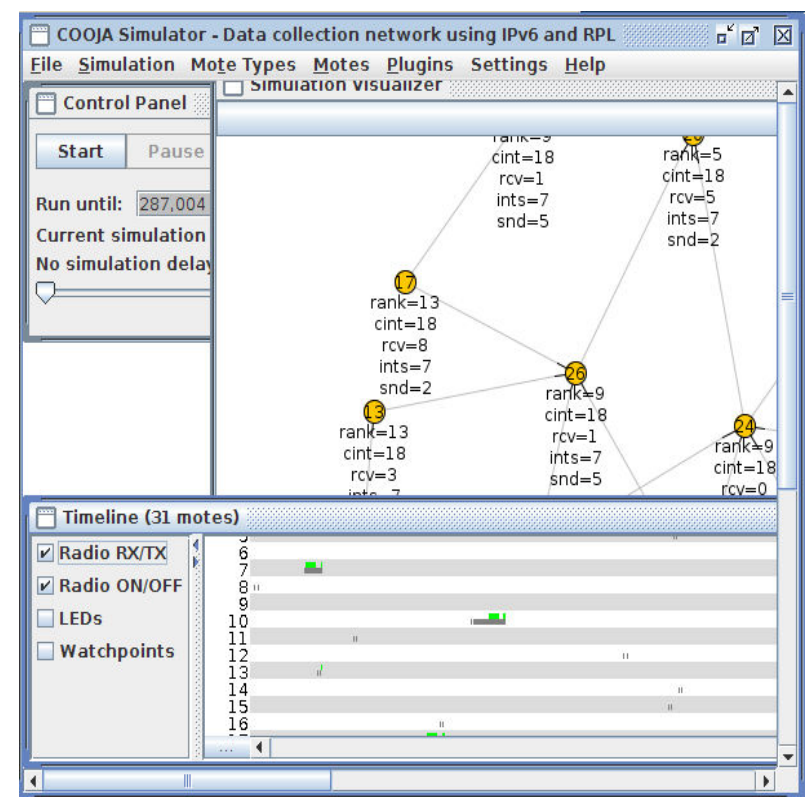

Figure 2: Screenshot of the Cooja/MSPsim lowpower network simulator showing the network and timeline visualizers.

ation in the Contiki operating system, the way by which new routing objective functions are added to ContikiRPL, and how the resulting system can be directly uploaded to a remote testbed consisting of Tmote Sky motes.

The demo shows the impact of choosing the routing objective function. One objective function uses a simple hop count and one uses expected transmissions (ETX) to do the forwarding decision. By running the two objective functions in the simulator and in a remote testbed, the simulation results and experimental measurements show that the simple hop-count objective function results in a shorter path length at the expense of higher power consumption.

\section{ACKNOWLEDGMENTS}

This work was partly financed by SSF, the Swedish Foundation for Strategic Research, through the Promos project.

\section{REFERENCES}

[1] M. Durvy, J. Abeillé, P. Wetterwald, C. O'Flynn, B. Leverett, E. Gnoske, M. Vidales, G. Mulligan, N. Tsiftes, N. Finne, and A. Dunkels. Making Sensor Networks IPv6 Ready. In Proceedings of ACM SenSys, Raleigh, North Carolina, USA, November 2008.

[2] J. Hui and D. Culler. IP is Dead, Long Live IP for Wireless Sensor Networks. In Proceedings of ACM SenSys, Raleigh, North Carolina, USA, November 2008.

[3] J.P. Vasseur and A. Dunkels. Interconnecting Smart Objects with IP: The Next Internet. Morgan Kaufmann, 2010.

[4] T. Winter (Ed.), P. Thubert (Ed.), and RPL Author Team. RPL: IPv6 Routing Protocol for Low power and Lossy Networks. Internet Draft draft-ietf-roll-rpl-09, work in progress. 